\title{
Continuous theta burst stimulation increases contralateral mu and beta rhythms with arm elevation
}

Citation for published version (APA):

Dionísio, A., Gouveia, R., Duarte, I. C., Castelhano, J., Duecker, F., \& Castelo-Branco, M. (2020).

Continuous theta burst stimulation increases contralateral mu and beta rhythms with arm elevation: implications for neurorehabilitation. Journal of Neural Transmission, 127(1), 17-25.

https://doi.org/10.1007/s00702-019-02117-6

Document status and date:

Published: 01/01/2020

DOI:

10.1007/s00702-019-02117-6

Document Version:

Publisher's PDF, also known as Version of record

Document license:

Taverne

Please check the document version of this publication:

- A submitted manuscript is the version of the article upon submission and before peer-review. There can be important differences between the submitted version and the official published version of record.

People interested in the research are advised to contact the author for the final version of the publication, or visit the DOI to the publisher's website.

- The final author version and the galley proof are versions of the publication after peer review.

- The final published version features the final layout of the paper including the volume, issue and page numbers.

Link to publication

\footnotetext{
General rights rights.

- You may freely distribute the URL identifying the publication in the public portal. please follow below link for the End User Agreement:

www.umlib.nl/taverne-license

Take down policy

If you believe that this document breaches copyright please contact us at:

repository@maastrichtuniversity.nl

providing details and we will investigate your claim.
}

Copyright and moral rights for the publications made accessible in the public portal are retained by the authors and/or other copyright owners and it is a condition of accessing publications that users recognise and abide by the legal requirements associated with these

- Users may download and print one copy of any publication from the public portal for the purpose of private study or research.

- You may not further distribute the material or use it for any profit-making activity or commercial gain

If the publication is distributed under the terms of Article $25 \mathrm{fa}$ of the Dutch Copyright Act, indicated by the "Taverne" license above, 


\title{
Continuous theta burst stimulation increases contralateral $m u$ and beta rhythms with arm elevation: implications for neurorehabilitation
}

\author{
Ana Dionísio ${ }^{1,2,3} \cdot$ Rita Gouveia $^{1} \cdot$ Isabel Catarina Duarte ${ }^{1,3} \cdot$ João Castelhano ${ }^{1,3} \cdot$ Felix Duecker $^{1,4,5}$. \\ Miguel Castelo-Branco ${ }^{1,3,6}$
}

Received: 21 September 2019 / Accepted: 7 December 2019 / Published online: 16 December 2019

(c) Springer-Verlag GmbH Austria, part of Springer Nature 2019

\begin{abstract}
The study of the physiological effects underlying brain response to transcranial magnetic stimulation is important to understand its impact on neurorehabilitation. We aim to analyze the impact of a transcranial magnetic stimulation protocol, the continuous theta burst (cTBS), on human neurophysiology, particularly on contralateral motor rhythms. cTBS was applied in 20 subjects over the primary motor cortex. We recorded brain electrical activity pre- and post-cTBS with electroencephalography both at rest and while performing motor tasks, to evaluate changes in brain oscillatory patterns such as $m u$ and beta rhythms. Moreover, we measured motor-evoked potentials before and after cTBS to assess its impact on brain's excitability. On the hemisphere contralateral to the protocol, we did observe a significant increase in $m u(p=0.027)$ and beta $(p=0.006)$ rhythms from pre- to post-cTBS, at the beginning of arm elevation. The topology of action planning and motor execution suggests that cTBS produced an inhibitory effect that propagated to the contralateral hemisphere, thereby precluding the expected/desired excitation for therapy purposes. This novel approach provides support for the notion that this protocol induces inhibitory changes in contralateral motor rhythms, by decreasing desynchronization, contradicting the ipsilateral inhibition vs. contralateral disinhibition hypothesis. Our results have implications for personalized cTBS usage as a rehabilitation intervention, suggesting that an unexpected propagation of inhibition can occur.
\end{abstract}

Keywords Neuromodulation · Plasticity · Continuous theta burst stimulation · Transcranial magnetic stimulation · Oscillation

\section{Introduction}

Transcranial magnetic stimulation (TMS) is a well-known non-invasive neuronal stimulation technique (Amassian and Maccabee 2006; Groppa et al. 2012) which has been applied to the study of several neurologic and psychiatric disorders, including the investigation of its therapeutic potential (George et al. 1999; Kobayashi and Pascual-Leone 2003; Chipchase et al. 2012; Heaton 2012).

Repetitive TMS allows the modulation of excitability for a period exceeding stimulation duration (Heaton 2012).

Ana Dionísio and Rita Gouveia have contributed equally to this work.

Miguel Castelo-Branco

mcbranco@fmed.uc.pt

Extended author information available on the last page of the article
Depending on the selected parameters, it can produce either excitatory or inhibitory modulation (Kobayashi and PascualLeone 2003; Rossi et al. 2009).

Despite its undoubted potential, large variability has actually been reported in terms of stimulation responses, even in which concerns response polarity (Heaton 2012), with some individuals showing inhibition and others excitation following the same protocol. Substantial effort is still required to understand the underlying brain responses, compare protocols and confirm their efficacy (Heaton 2012).

Here, we aimed to analyze a TMS protocol, seeking to unravel its effects on brain activity and complex network responses, as expressed by cortical oscillatory responses. In this study, we focus on continuous theta burst stimulation (cTBS), a patterned form of repetitive TMS first proposed by Huang et al. (Huang et al. 2005), where 3 pulses at $50 \mathrm{~Hz}$ are applied every $200 \mathrm{~ms}(5 \mathrm{~Hz})$ for a total duration of $40 \mathrm{~s}$ (Sandrini et al. 2011). The cTBS protocol is an 
inhibitory protocol with the effect lasting up to $1 \mathrm{~h}$ when it is performed for $40 \mathrm{~s}$ (Sandrini et al. 2011). Still, the variability of the responses, including duration of the effects, associated with other repetitive TMS protocols has also been repeatedly reported by several authors investigating cTBS applications (Jannati et al. 2017; Lowe and Hall 2018; Rocchi et al. 2018).

It is widely accepted that motor brain activity is controlled between hemispheres in a task-dependent manner, in healthy individuals. This push-pull mechanism is believed to occur through interhemispheric inhibition. Some pathologies can affect such control of activity across hemispheres. TMS can be used as a therapeutic strategy, by applying high or low frequencies to increase or decrease the excitability on the stimulated hemisphere, respectively, with the goal to restore normal activity patterns (Dionísio et al. 2018). Some theories postulate that hemispheric inhibition can help to boost activity in the contralateral (lesioned) hemisphere. Therefore, it remains an important question whether the repetitive TMS protocols can affect not only the stimulation site but also the contralateral areas, through interhemispheric connections, and whether this leads to increased/ decreased activity in the contralateral hemisphere. To test this hypothesis, we applied cTBS to healthy volunteers to evaluate its impact on human neurophysiology, and to test its contralateral effects. A general a priori expectation would be that such effects should be excitatory, through disinhibition due to ipsilateral inhibition, but this remains controversial. It is also important to recognize that most studies just look at motor-evoked potentials (MEPs); whereas, local and remote EEG effects of cTBS in the context of a motor task are rarely investigated and might be more complex than expected. Actually, Rocchi et al. (Rocchi et al. 2018) point out the possibility of MEP's amplitude information being incomplete and not reflecting all cortical outputs. It has been reported that action planning and motor execution involve different processing mechanisms, indexed by distinct frequency bands (in intervals such as 8-10, 10-12 and 15-25 Hz) (Pfurtscheller and Lopes Da Silva 1999; Pfurtscheller et al. 2000; Pineda 2005; Ilmoniemi and Kičić 2010; Ramos-Murguialday and Birbaumer 2015). Therefore, we studied cortical oscillatory patterns (namely $m u$ and beta rhythms) with electroencephalography (EEG), before and after the stimulation. We aimed to study if the stimulation protocol affects contralateral motor rhythms, as hypothesized according to the above-mentioned ipsilateral inhibition vs. contralateral disinhibition hypothesis. We recorded electrical cerebral activity at rest to evaluate the physiological state and during motor execution to study brain oscillatory patterns (event-related synchronization (ERS) and desynchronization (ERD)). Increased synchronization and amplitude of such oscillatory patterns reflect idle states characterized by inhibited processing of visual information, somatosensory input or motor output, as a response to a stimulus or event (Pfurtscheller et al. 1996, 1997). These inhibition-related rhythms are referred to as occipital alpha rhythm, for visual areas, or central $m u$ rhythm or beta, concerning sensorimotor areas (Pfurtscheller et al. 1996, 1997). However, these rhythms can be blocked in an activated state (such as in motor readiness/movement preparation), where cortical neurons are excited and neuronal assemblies are likely to work individually, by a phenomenon described as ERD (Pfurtscheller et al. 1997). The extent of ERD is proportional to the extent into which neural networks are recruited during the execution of a given task (Pfurtscheller et al. 1996). On the other hand, the resting or idling of those areas is associated with a decrease of excitability or inhibition of neuronal populations which remain in a more synchronous mode (Pfurtscheller et al. 1997). Therefore, such large-scale idle synchrony, translates into an ERS (Pfurtscheller et al. 1997). Motor-evoked potentials, quantified by electromyography (EMG), were also evaluated, as a measure of motor cortex output.

To the best of our knowledge, this is the first study that so far assesses the effects of cTBS applied to the primary motor cortex, using both EMG for measuring motor output and EEG for studying brain oscillation patterns during motor tasks in healthy subjects, to predict physiological effects and their implications for neurorehabilitation. We found an increase in motor rhythms ( $m u$ and beta) of the non-stimulated hemisphere, after continuous theta burst stimulation, which we interpret as an indication that inhibitory ipsilateral effects might actually propagate to the contralateral hemisphere instead of the prediction raised by the ipsilateral inhibition vs. contralateral disinhibition hypothesis.

\section{Materials and methods}

This study was approved by the Ethics Committee of the Faculty of Medicine of the University of Coimbra and was conducted according to the Declaration of Helsinki. We explained the experimental procedure and obtained the written informed consent from all participants.

\section{Sample}

To enroll the study, subjects should (1) be aged between 40 and 85 years, and (2) have the capability to understand the tasks. Exclusion criteria included (1) history of neurological disorders, (2) motor deficits, (3) cognitive impairment, (4) diagnosed dementia, (5) history of epilepsy, (6) drug or alcohol abuse, and (7) contraindications to transcranial magnetic stimulation, as assessed by a questionnaire based on published guidelines (Rossi et al. 2009, 2011). 
We included 20 right-handed healthy volunteers who fulfilled the eligibility criteria. After enrolment, subjects were randomized in a 1:1 ratio to receive the cTBS protocol on the right $(\mathrm{RH})$ or left $(\mathrm{LH})$ hemisphere. Table 1 presents the demographic data of the subjects. Participants' age ranged between 41 and 75 years, with a mean of $61.50 \pm 11.965$ years for the RH group and of $58.90 \pm 10.939$ years for the LH group.

Table 1 Demographic data of volunteers

\begin{tabular}{ll}
\hline & $\begin{array}{l}\text { Total of } \\
\text { participants } \\
(N=20)\end{array}$ \\
\hline Age (years; mean $\pm \mathrm{SD})$ & $60.20 \pm 11.237$ \\
Gender (female/male) & $11 / 9$ \\
Handedness (points; mean $\pm \mathrm{SD})^{\mathrm{a}}$ & $35.75 \pm 0.550$ \\
Education (years; mean $\pm \mathrm{SD})$ & $15.00 \pm 2.938$ \\
\hline
\end{tabular}

$S D$ standard deviation

${ }^{\text {a }}$ The maximum score for handedness, assessed by an adapted Edinburgh Handedness Inventory questionnaire, was 36 points
Figure 1 presents the experimental design, showing the order of procedures and the selection of the electrodes for electroencephalography.

\section{Electroencephalography (EEG) task}

Before the acquisition, we explained to the participant the sequence of the motor paradigm. Then, we used "GO" and "STOP" commands to ask subjects to begin and stop the movement, respectively. Task commands were marked with triggers in the EEG file during the online recording. At the beginning, to study physiological activity at rest, including posterior alpha patterns, subjects had to open and close the eyes nine times, maintaining the eyes opened/closed for $10 \mathrm{~s}$ each trial. Subsequently, individuals were asked to perform two different motor tasks (simple and complex, the latter requiring coordinated sequential and paced movements, in addition to the initiation, maintenance and stop phases) to evaluate electrophysiological motor responses. The first task consisted of $90^{\circ}$-arm elevation. Each individual was instructed to move the arm up, to sustain the movement for $15 \mathrm{~s}$ and then move the arm down. Each movement was repeated 6 times. The second task was a more complex
Fig. 1 Experimental design and procedures. $\mathrm{RH}$ and $\mathrm{LH}$ represent the participants who received the continuous theta burst stimulation protocol on the right and on the left hemisphere, respectively

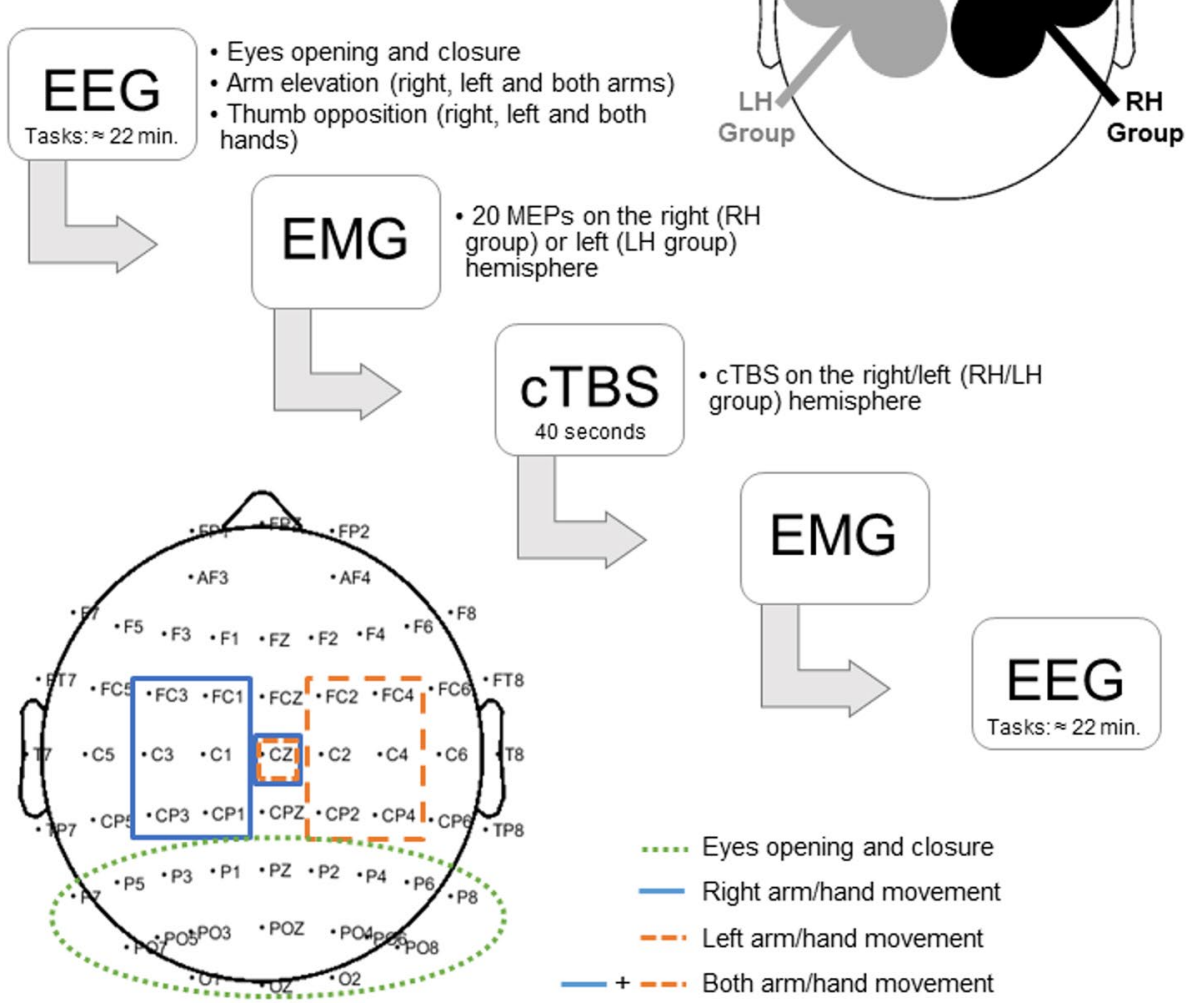


movement, named thumb opposition, where the subject had to touch with the thumb in each one of the remaining fingers sequentially until receiving the "STOP" command. Fifteen seconds after, they had to repeat the task during $15 \mathrm{~s}$ more. This procedure was also performed six times. The movements were executed first with the right arm/hand, then with the left and at last with both limbs simultaneously (6 times each).

\section{EEG data acquisition and analysis}

Brain electrical activity was recorded before and after the stimulation protocol, using a SynAmps2 RT amplifier and Scan 4.5 software (Compumedics, Charlotte, NC). We used an EEG cap with 64 electrodes (QuickCap, NeuroScan, USA) placed according to the International 10-20 system. Skin preparation was performed with abrasive gel and alcohol at $96 \%$ to maintain electrode impedances under $10 \mathrm{k} \Omega$. We selected an acquisition sampling rate of $1000 \mathrm{~Hz}$ and added a low-pass filter at $200 \mathrm{~Hz}$ and the signal was highpassed from the DC level. The EEG signal was processed with Scan 4.5 software (Compumedics, Charlotte, NC) and EEGLAB v.14.1.1b (Delorme and Makeig 2004), an interactive MATLAB toolbox. We filtered the signal offline, from 1 to $45 \mathrm{~Hz}$, and then down-sampled data from 1000 to $250 \mathrm{~Hz}$. The average re-reference approach was used. Muscle artefacts were checked by visual inspection and eliminated.

Independent Component Analysis (ICA) was computed and components including eye movements and blinks were removed. In any case, prior to removal, the rate of occurrence of these artefacts was similar between pre- and post-stimulation conditions, for both tasks (arm elevation: $t=-0.029, p=0.977$; thumb opposition: $t=1.259$, $p=0.223$ ). For data processing, the rest condition was used as a baseline.

Custom MATLAB (version R2017b, The MathWorks, USA) scripts were implemented [adapted from our previous works by Castelhano et al.(Castelhano et al. 2013) and by Silva et al. (Silva et al. 2016)] for the pre-processing and power quantification. Briefly, we conducted time-frequency analysis as reported by Uhlhaas et al. (2006) and other works (Lachaux et al. 1999; Rodriguez et al. 1999; Uhlhaas et al. 2006; Melloni et al. 2007; Castelhano et al. 2013), by applying the pseudo Wigner-Ville transformation. The amplitude and phase were computed throughout all time window and frequency bin (resolution of $1 \mathrm{~Hz} /$ frequency bin), from 5 to $40 \mathrm{~Hz}$, in steps of $1 \mathrm{~Hz}$, specifically in the visual alpha, $m u$ and beta frequency bands and in the time range of the epochs defined below. This analysis was performed within the selected electrode clusters (Fig. 1).

Quantification of alpha power $(8-13 \mathrm{~Hz}$ ) for the eyes' closure and opening control conditions was performed between -2000 and 10,000 ms, with the epochs of the baseline being defined between -2000 and $0 \mathrm{~ms}$. Quantification of $m u$ rhythm $(10-12 \mathrm{~Hz})$ and beta power $(15-25 \mathrm{~Hz})$ for the motor tasks was carried out from -2000 to $0 \mathrm{~ms}$ (pre-movement and preparation) and from 0 to $4000 \mathrm{~ms}$ (time-locked to the beginning of the movement), where the period from -2000 to $-1500 \mathrm{~ms}$ was assigned as baseline.

Scalp ERS/ERD mapping of $m u$ and beta rhythms was performed with eConnectome (He et al. 2011), using default parameters.

\section{Electromyography (EMG)}

Motor-evoked potentials were recorded with surface electromyography, using the BIOPAC MP-150 system and the EMG 100C amplifier (Biopac Systems, CA, USA), with a gain of $1000 . \mathrm{Ag} / \mathrm{AgCl}$ electrodes were placed in a bellytendon montage. We used the Acqknowledge 4.2 software (Biopac Systems, CA, USA) to record the EMG signal at a $2.500 \mathrm{kHz}$ sampling rate, and then to determine the peak-topeak amplitude of the MEPs, trial-by-trial.

\section{Transcranial magnetic stimulation (TMS)}

Transcranial magnetic stimulation was performed with MagPro X100 magnetic stimulator connected to a 70-mm Fig-of-eight coil (MagVenture, Denmark). Participants wore earplugs and were comfortably seated in an armchair during the experiment.

We placed the coil at the hotspot over the primary motor cortex area, at $45^{\circ}$ to the sagittal plane and assessed the resting motor threshold. Then, we selected the intensity that produced motor-evoked potentials with a peak-to-peak amplitude between 0.50 and $1 \mathrm{mV}$, and recorded 20 MEPs to establish a baseline measurement.

The repetitive protocol was administered on the same hemisphere, right or left, according to the randomization procedure. The active motor threshold was measured as the minimum intensity required to observe at least one minimal muscle twitch on the hand in three trials, while the upper limbs were performing an isometric contraction. This value was used as the intensity for the cTBS protocol. The protocol lasted $40 \mathrm{~s}$ and was administered as previously described on the literature: with 3 pulses at $50 \mathrm{~Hz}$ being applied every $200 \mathrm{~ms}$, resulting in a total of 600 pulses (Huang et al. 2005; Sandrini et al. 2011).

To assess differences on the brain's excitability induced by the stimulation, we repeated the MEPs' peak-to-peak amplitude measurement post-cTBS, with the same intensity used before the cTBS protocol. This measure was taken 5 min after the end of the protocol, when the largest effects are thought to take place (Di Lazzaro et al. 2005; Chung et al. 2016). 
After the cTBS protocol all data, including EMG and EEG, were recorded within the 1-h window, complying with the duration of the neurophysiological effects (Sandrini et al. 2011).

\section{Statistical analysis}

The statistical analysis of the data was performed on the SPSS Statistics software, version 24 (IBM SPSS Statistics, IBM Corporation, Chicago, IL). We adopted a confidence interval of $95 \%$. Normality of data was assessed by the Shapiro-Wilk Test. Differences in the occurrence of EEG artefacts associated with eye movements and blinks were evaluated with Paired $t$ student test. To study intergroup differences in demographic data, we used the Mann-Whitney $U$ test for age, handedness and education, and Fisher's exact test, for gender. The Wilcoxon test was applied to evaluate the effects of the cTBS protocol, comparing the post- to the pre-stimulation measures.

\section{Results}

There were no statistically significant differences between subgroups concerning age $(U=40.500, p=0.490)$, gen$\operatorname{der}(p=1.000)$, handedness assessed by an adapted Edinburgh Handedness Inventory questionnaire (Oldfield 1971) ( $U=39.500, p=0.458)$ or education $(U=37.000, p=0.336)$. Severe adverse events were not observed.

\section{Motor-evoked potentials}

We found a bimodal effect of the protocol, with some patients showing an amplitude increase and the others revealing a decrease (positive and negative peaks, with scarce near null responses). Indeed, the direction of the effects was balanced across participants, with $40 \%$ of subjects exhibiting an increase of the peak-to-peak amplitude of the MEPs on the stimulated hemisphere. Differences between pre- and post-cTBS were not statistically significant, in line with the bimodal effect $(Z=-0.511, p=0.639)$.

\section{Electroencephalography}

Differences in visual alpha power between pre- and postcTBS were, as expected, not significant in the control condition, either when the subjects had the eyes opened $(Z=-0.933, p=0.368)$ or closed $(Z=-0.597, p=0.571)$.

Concerning the motor rhythms, we found significant differences in power between pre- and post-cTBS. Changes are visible in the topography maps shown in Fig. 2 (example participant allocated to the $\mathrm{RH}$ group), during the beginning of arm elevation for both $m u$ and beta rhythms, pre- and post-stimulation. These maps illustrate notable changes induced by the protocol, showing an interesting inversion of the signal polarity after cTBS and a bilateral mirror symmetric activity pattern in central areas (suggesting similar effects across hemispheres).

The above-mentioned differences in the $m u$ band were significant for the simple arm elevation task (and not the complex thumb opposition), on the hemisphere contralateral to the protocol, both for pre-movement and preparation $(Z=-1.979, p=0.048$, Fig. 3a) and when beginning elevation $(Z=-2.203, p=0.027$, Fig. 3a, b). These were translated into a significant increase of the mean $т и$ power on the contralateral hemisphere, after stimulation. Similarly, regarding beta power, we observed a significant increase between pre- and post-cTBS on the hemisphere contralateral to the protocol, when beginning arm elevation $(Z=-2.688$, $p=0.006$, Fig. 3c, d). No significant differences were found for the beta rhythm on pre-movement and preparation.

\section{Discussion}

In this study, we found a bimodal effect of the cTBS protocol in terms of motor-evoked responses as indicators of modulation of excitability and inhibition, and more consistent effects in motor related rhythms, which reflect also non-idle versus idle states. It is well known that increases in power of $m u$ oscillations (ERS) reflect a motor inhibitory effect and a more idle state, which also occurs for alpha and beta oscillations (Jannati et al. 2017). The latter was the most important finding of this study. Concerning the bimodal pattern in motor-evoked potentials, a similar effect was described by Hamada et al. (Hamada et al. 2013). According to those authors, it could have been caused by differences in the recruitment of cortical neurons, as suggested by changes observed when the MEP's latency was analyzed (Hamada et al. 2013). Moreover, Rocchi et al. (Rocchi et al. 2018) also reported conflicting results, since they observed a facilitation of MEPs or no effect at all, after the application of cTBS. It might be that some participants are non-responders, and in those cases cTBS effects are not observed (Lowe and Hall 2018). However, we cannot disregard that the intensity for the application of the cTBS protocol was here selected as a function of the active motor threshold, as it is usual practice, with this measure being established following a voluntary muscle contraction. It is possible that the activation of the target muscle before, during or after stimulation could influence the modulation of plasticity, being able to cancel or even invert the effects detected by motor-evoked potentials (Goldsworthy et al. 2014; Opie et al. 2017). In fact, it is known that a higher number of descending volleys and a reduction of spinal motoneurons threshold do occur following muscular activation pre-stimulation (Lepage et al. 2008). 


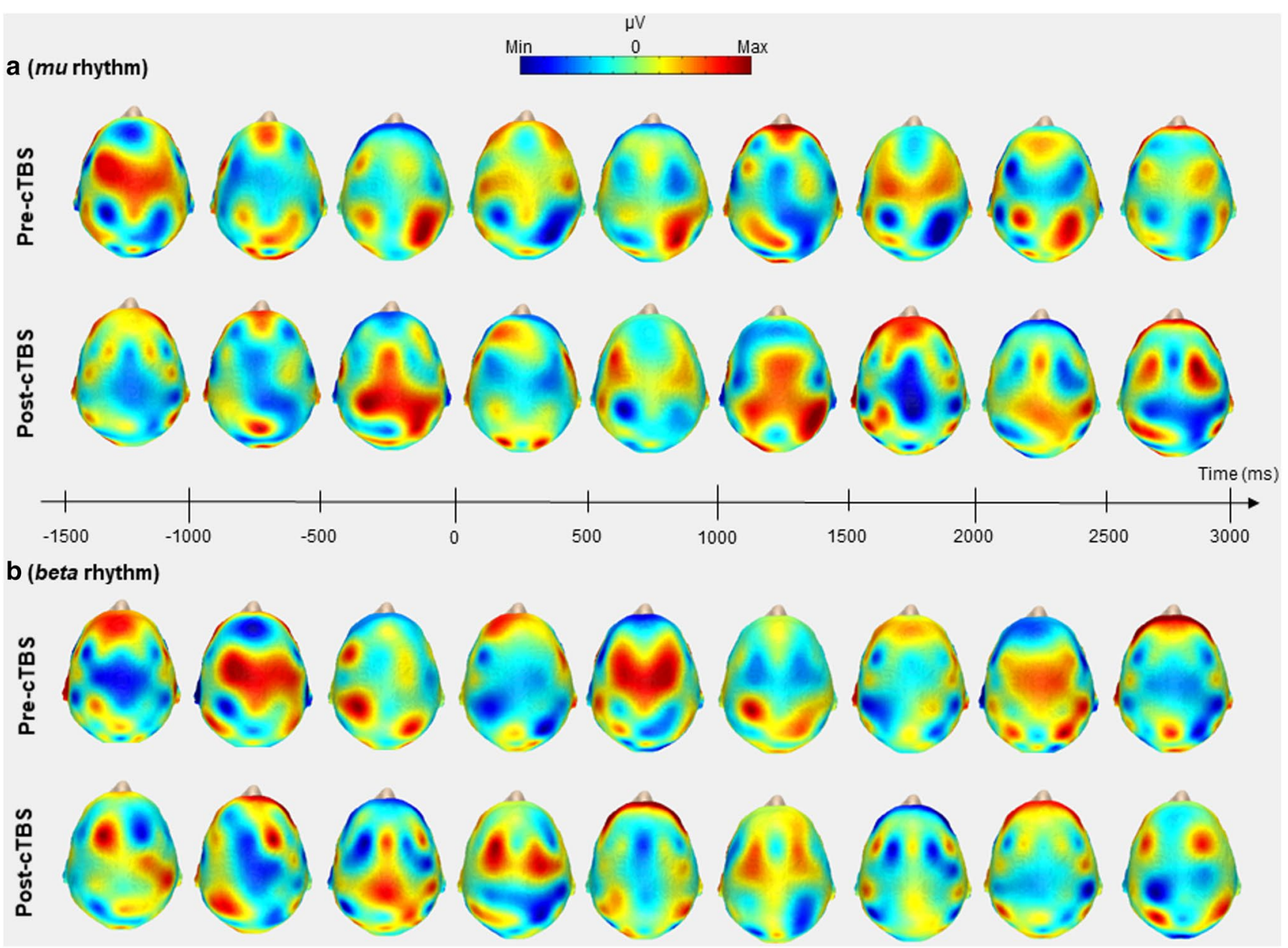

Fig. 2 Example of scalp ERS/ERD mapping. $M u$ a and beta $\mathbf{b}$ rhythms are presented from a participant who received continuous theta burst stimulation on the right hemisphere, during elevation of the arm ipsilateral to the stimulation, pre- and post-cTBS. Each scalp map represents the mean of ERS/ERD on the corresponding time period
At the same time, some authors suggest that MEPs may not express all cortical outputs, reflecting exclusively those destined to the spinal cord (Rocchi et al. 2018). Although both MEPs and EEG are considered reliable, and although the amplitude of motor-evoked potentials is, in part, influenced by spinal processes or other events remote to the brain, these are not expected to affect EEG data (Lepage et al. 2008; Rocchi et al. 2018). In this concern, our EEG recordings, and the nature of their neural sources are much less susceptible to be influenced by these types of effects.

The arm elevation task showed significant differences between pre- and post-intervention in the contralateral hemisphere. This is potentially interesting in terms of potential application in neurorehabilitation, because it was possible to detect an effect of the protocol on the $m u$ and beta rhythms. This effect was specifically observed in a mirror symmetric way on the hemisphere contralateral to the protocol, possibly reflecting the topology of action planning and execution. The robustness of this finding could be attributed to the fact that there was less inter-subject variability on the contralateral hemisphere's response to cTBS. Actually, in this hemisphere, the majority of the volunteers showed a power increase in $т и$ (13 out of 20) and beta (16 out of 20) rhythms, when beginning arm elevation after stimulation. On the pre-stimulation condition, we observed the expected event-related desynchronization when the participant was performing the movement, translating to negative values for the power $(\mathrm{dB})$, comparing to the baseline. After cTBS, the power increased on the contralateral hemisphere, becoming less negative. The protocol decreased the desynchronization (in other words more relative synchronization). This increase in power suggests an inhibitory response on the sensorimotor cortical areas associated with an event-related synchronization. Moreover, on the corresponding topographic maps, we observed an inversion of the signal polarity after the application of continuous theta burst stimulation, with both hemispheres responding in a more congruent manner. Hence, cTBS was 

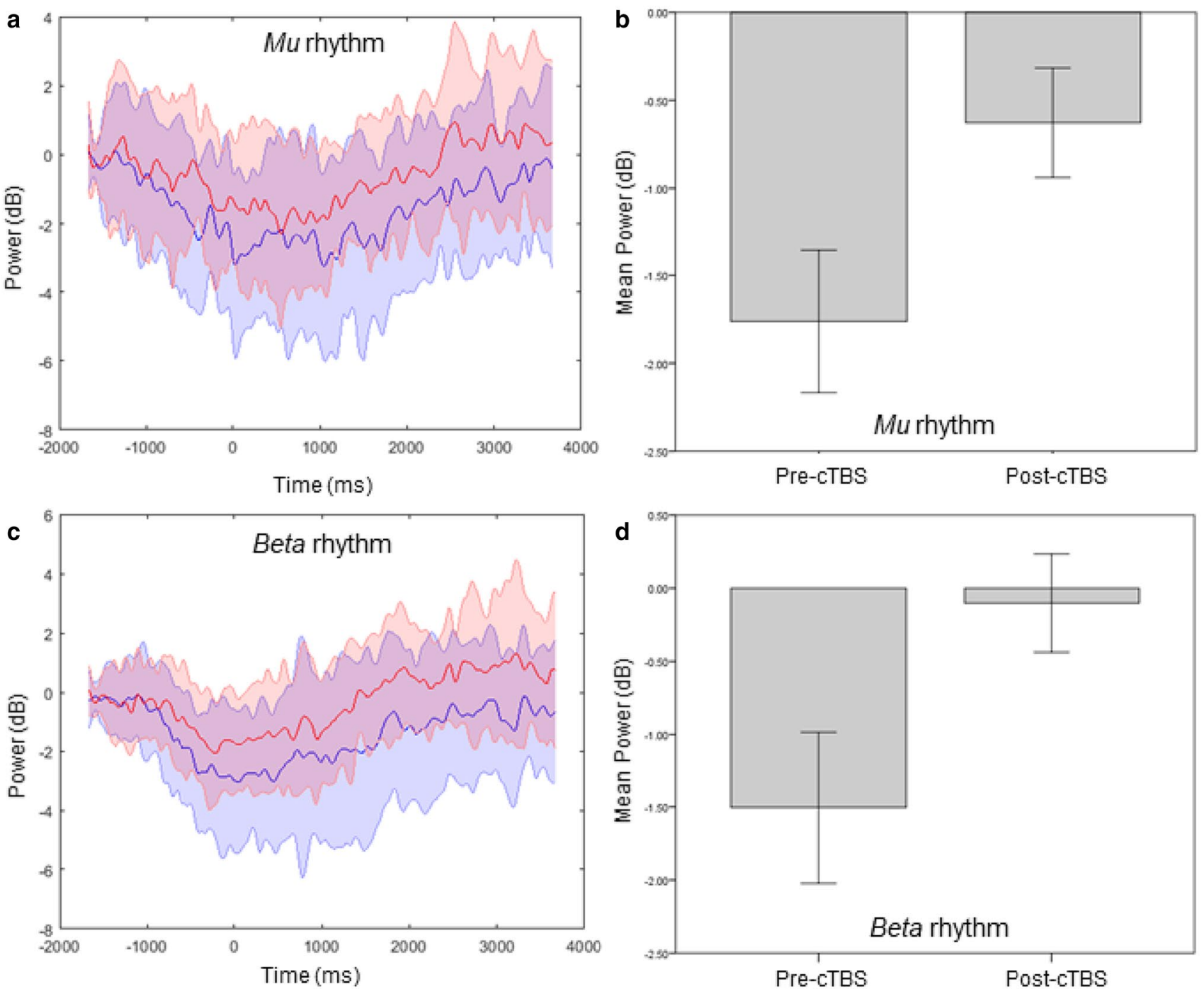

Fig. 3 Time-response plots showing changes in $m u$ a and beta c rhythms following cTBS, during pre-movement and preparation, and beginning of the arm elevation, on the hemisphere contralateral to the protocol. Blue represents pre-cTBS and red illustrates post-cTBS

able to influence the brain's neurophysiology by inducing a change in motor cortex that propagated to the contralateral hemisphere, corroborating our hypothesis that the protocol affects the contralateral motor rhythms. However, this change is not in the opposite direction, as expected in our initial hypothesis, but instead congruent with the manipulation in the ipsilateral hemisphere. In other words, the spreading of inhibition would render this protocol problematic for therapeutic modulation of a lesioned contralateral hemisphere. Other interpretations are, however, possible, namely nonlinear effects of artificial stimulation on signal- to- noise levels. This result substantiates the need for future studies that further analyze the electrophysiological changes underlying brain responses to the continuous theta burst protocol.

data. The significant differences $(p$ value $<0.05)$ on mean power of $m u \mathbf{b}$ and beta $\mathbf{d}$ rhythms for the beginning of the arm elevation are also depicted. Error bars represent $\pm 1 \mathrm{SE}$

Remarkably, we did observe significant protocol-related differences only for the arm elevation task and not for the more fine-tuned thumb opposition, possibly because the former involves simpler motor programs that can be more easily targeted with our protocol. Also, it has been reported that more complex tasks lead augmented effort and attentional effects known to modulate ERDs (Pfurtscheller et al. 1996; Pfurtscheller and Lopes Da Silva 1999), which might cancel other effects.

In this study, EEG was more sensitive to detect neural modulation changes than EMG. It was already suggested in the literature that EEG can help in clarifying the effects of TMS (Rocchi et al. 2018). We suggest that this finding can be related to differences in the intrinsic variability of both techniques. It has been reported that the combination 
of TMS with EEG provides evoked responses that are less variable and more consistent, in comparison with MEPs (Ilmoniemi and Kičić 2010; Rocchi et al. 2018), considering that EEG measures, such as oscillations, are not affected by spinal cord excitability (Rocchi et al. 2018). Moreover, we chose to measure brain rhythms with relatively high signal-to-noise ratios (alpha, mu and beta) involved in either sensory or motor responsiveness. Our results suggest that it would be valuable to explore the potential of EEG in evaluating brain responses to TMS protocols, by including this technique on the design of future studies.

\section{Conclusions}

As far as we are aware, this is a novel study on the evaluation of the physiological effects of continuous theta burst stimulation applied to the primary motor cortex, which measures motor-evoked potentials with EMG and $m u$ and beta rhythms with EEG during simple and complex motor execution tasks, in healthy subjects. EEG showed more reliable and consistent effects than MEPs recordings. Our findings suggest that the cTBS protocol changes brain's neurophysiology, by decreasing the desynchronization of the contralateral $m u$ and beta rhythms, with a direct impact on increased synchronization levels and inhibition. This propagation of inhibition has strong implications for the design of neurorehabilitation protocols, since it shows that contralateral excitation of a lesioned hemisphere might not occur following the application of an inhibitory protocol in the healthy ipsilateral hemisphere.

Acknowledgements We want to thank Ana Cristina Vidal for helping on the selection of the EEG tasks. Moreover, we want to acknowledge Gilberto Silva for his help on EEG data analysis and Francisco Oliveira for his statistical support. We thank all volunteers for their essential collaboration.

Funding This project has received funding from the European Union's Horizon 2020 research and innovation program under the Marie Sklodowska-Curie grant agreement No. 708492 (FD), from Fundação Luso-Americana para o Desenvolvimento [Prémio FLAD Life Sciences 2020] and Portuguese Foundation for Science and Technology (FCT), COMPETE, POCI-01-0145-FEDER-007440, FCT-UID/NEU/04539/2013, BIGDATIMAGE, CENTRO-01-0145FEDER-000016 financed by Centro 2020 FEDER, COMPETE, PACMEDPERSYST POCI-01-0145- FEDER-016428. The funders had no role in study design, data collection and analysis, decision to publish, or preparation of the manuscript.

\section{Compliance with ethical standards}

Conflict of interest The authors declare that they have no conflict of interest.

\section{References}

Amassian VE, Maccabee PJ (2006) Transcranial magnetic stimulation. Conf Proc IEEE Eng Med Biol Soc 1:1620-1623. https:// doi.org/10.1109/IEMBS.2006.259398

Castelhano J, Rebola J, Leitão B et al (2013) To perceive or not perceive: the role of gamma-band activity in signaling object percepts. PLoS One. https://doi.org/10.1371/journal.pone.0066363

Chipchase L, Schabrun S, Cohen L et al (2012) A checklist for assessing the methodological quality of studies using transcranial magnetic stimulation to study the motor system: an international consensus study. Clin Neurophysiol 123:1698-1704. https://doi.org/10.1016/j.clinph.2012.05.003

Chung SW, Hill AT, Rogasch NC et al (2016) Use of theta-burst stimulation in changing excitability of motor cortex: a systematic review and meta-analysis. Neurosci Biobehav Rev 63:43-64

Delorme A, Makeig S (2004) EEGLAB: an open source toolbox for analysis of single-trial EEG dynamics including independent component analysis. J Neurosci Methods 134:9-21. https://doi. org/10.1016/j.jneumeth.2003.10.009

Di Lazzaro V, Pilato F, Saturno E et al (2005) Theta-burst repetitive transcranial magnetic stimulation suppresses specific excitatory circuits in the human motor cortex. J Physiol 565:945-950. https://doi.org/10.1113/jphysiol.2005.087288

Dionísio A, Duarte IC, Patrício M, Castelo-Branco M (2018) The use of repetitive transcranial magnetic stimulation for stroke rehabilitation: a systematic review. J Stroke Cerebrovasc Dis 27:131. https://doi.org/10.1016/j.jstrokecerebrovasdis.2017.09.008

George MS, Lisanby SH, Sackeim HA (1999) Transcranial magnetic stimulation: applications in neuropsychiatry. Arch Gen Psychiatry 56:300-311. https://doi.org/10.1001/archpsyc.56.4.300

Goldsworthy MR, Müller-Dahlhaus F, Ridding MC, Ziemann U (2014) Inter-subject variability of LTD-like plasticity in human motor cortex: a matter of preceding motor activation. Brain Stimul 7:864-870. https://doi.org/10.1016/j.brs.2014.08.004

Groppa S, Oliviero A, Eisen A et al (2012) A practical guide to diagnostic transcranial magnetic stimulation: report of an IFCN committee. Clin Neurophysiol 123:858-882. https://doi. org/10.1016/j.clinph.2012.01.010

Hamada M, Murase N, Hasan A et al (2013) The role of interneuron networks in driving human motor cortical plasticity. Cereb Cortex 23:1593-1605. https://doi.org/10.1093/cercor/bhs147

He B, Dai Y, Astolfi L et al (2011) EConnectome: a MATLAB toolbox for mapping and imaging of brain functional connectivity. J Neurosci Methods 195:261-269. https://doi.org/10.1016/j. jneumeth.2010.11.015

Heaton J (2012) Repetitive transcranial magnetic stimulation: a comparative trial of low frequency treatments. Thesis, Bachelor of Medical Science with Honours. University of Otago. Retrieved from http://hdl.handle.net/10523/2452

Huang YZ, Edwards MJ, Rounis E et al (2005) Theta burst stimulation of the human motor cortex. Neuron 45:201-206. https:// doi.org/10.1016/j.neuron.2004.12.033

Ilmoniemi RJ, Kičić D (2010) Methodology for combined TMS and EEG. Brain Topogr 22:233-248

Jannati A, Block G, Oberman LM et al (2017) Interindividual variability in response to continuous theta-burst stimulation in healthy adults. Clin Neurophysiol 128:2268-2278. https://doi. org/10.1016/j.clinph.2017.08.023

Kobayashi M, Pascual-Leone A (2003) Transcranial magnetic stimulation in neurology. Lancet Neurol 2:145-156. https://doi. org/10.1016/S1474-4422(03)00321-1

Lachaux JP, Rodriguez E, Martinerie J, Varela FJ (1999) Measuring phase synchrony in brain 
signals. Hum Brain Mapp 8:194-208. https://doi.org/10.1002/ (SICI) 1097-0193(1999)8:4<194::AID-HBM4>3.0.CO;2-C

Lepage JF, Saint-Amour D, Théoret H (2008) EEG and neuronavigated single-pulse TMS in the study of the observation/execution matching system: are both techniques measuring the same process? J Neurosci Methods. https://doi.org/10.1016/j.jneum eth.2008.07.021

Lowe CJ, Hall PA (2018) Reproducibility and sources of interindividual variability in the responsiveness to prefrontal continuous theta burst stimulation (cTBS). Neurosci Lett 687:280-284. https ://doi.org/10.1016/j.neulet.2018.09.039

Melloni L, Molina C, Pena M et al (2007) Synchronization of neural activity across cortical areas correlates with conscious perception. J Neurosci. https://doi.org/10.1523/jneurosci.4623-06.2007

Oldfield RC (1971) The assessment and analysis of handedness: the Edinburgh inventory. Neuropsychologia 9:97-113. https://doi. org/10.1016/0028-3932(71)90067-4

Opie GM, Vosnakis E, Ridding MC et al (2017) Priming theta burst stimulation enhances motor cortex plasticity in young but not old adults. Brain Stimul 10:298-304. https://doi.org/10.1016/j. brs.2017.01.003

Pfurtscheller G, Stancák A, Neuper C (1996) Event-related synchronization (ERS) in the alpha band-an electrophysiological correlate of cortical idling: a review. Int J Psychophysiol 24:39-46. https:// doi.org/10.1016/S0167-8760(96)00066-9

Pfurtscheller G, Neuper C, Andrew C, Edlinger G (1997) Foot and hand area mu rhythms. Int J Psychophysiol 26:121-135

Pfurtscheller G, Lopes Da Silva FH (1999) Event-related EEG/MEG synchronization and desynchronization: basic principles. Clin Neurophysiol 110:1842-1857

Pfurtscheller G, Neuper C, Krausz G (2000) Functional dissociation of lower and upper frequency mu rhythms in relation to voluntary limb movement. Clin Neurophysiol 111:1873-1879. https://doi. org/10.1016/S1388-2457(00)00428-4

Pineda J (2005) The functional significance of mu rhythms: translating "seeing" and "hearing" into "doing". Brain Res Rev 50:57-68
Ramos-Murguialday A, Birbaumer N (2015) Brain oscillatory signatures of motor tasks. J Neurophysiol 113:3663-3682. https://doi. org/10.1152/jn.00467.2013

Rocchi L, Ibáñez J, Benussi A et al (2018) Variability and predictors of response to continuous theta burst stimulation: a TMS-EEG study. Front Neurosci. https://doi.org/10.3389/fnins.2018.00400

Rodriguez E, George N, Lachaux JP et al (1999) Perception's shadow: long-distance synchronization of human brain activity. Nature. https://doi.org/10.1038/17120

Rossi S, Hallett M, Rossini PM et al (2009) Safety, ethical considerations, and application guidelines for the use of transcranial magnetic stimulation in clinical practice and research. Clin Neurophysiol 120:2008-2039. https://doi.org/10.1016/j.clinp h.2009.08.016

Rossi S, Hallett M, Rossini PM, Pascual-Leone A (2011) Screening questionnaire before TMS: an update. Clin Neurophysiol 122:1686

Sandrini M, Umiltà C, Rusconi E (2011) The use of transcranial magnetic stimulation in cognitive neuroscience: a new synthesis of methodological issues. Neurosci Biobehav Rev 35:516-536. https ://doi.org/10.1016/j.neubiorev.2010.06.005

Silva G, Ribeiro MJ, Costa GN et al (2016) Peripheral attentional targets under covert attention lead to paradoxically enhanced alpha desynchronization in neurofibromatosis type 1 . PLoS One. https ://doi.org/10.1371/journal.pone.0148600

Uhlhaas PJ, Linden DEJ, Singer W et al (2006) Dysfunctional longrange coordination of neural activity during gestalt perception in schizophrenia. J Neurosci. https://doi.org/10.1523/jneur osci.2002-06.2006

Publisher's Note Springer Nature remains neutral with regard to jurisdictional claims in published maps and institutional affiliations.

\section{Affiliations}

\section{Ana Dionísio $^{1,2,3} \cdot$ Rita Gouveia $^{1}$ - Isabel Catarina Duarte ${ }^{1,3} \cdot$ João Castelhano ${ }^{1,3} \cdot$ Felix Duecker $^{1,4,5}$. Miguel Castelo-Branco ${ }^{1,3,6}$ (D)}

1 Coimbra Institute for Biomedical Imaging and Translational Research (CIBIT), Institute of Nuclear Sciences Applied to Health (ICNAS), University of Coimbra, Azinhaga de Santa Comba, 3000-548 Coimbra, Portugal

2 Department of Physics, Faculty of Sciences and Technology, University of Coimbra, Coimbra, Portugal

3 Institute for Biomedical Imaging and Life Sciences (CNC.IBILI), Faculty of Medicine, University of Coimbra, Coimbra, Portugal
4 Department of Cognitive Neuroscience, Faculty of Psychology and Neuroscience, Maastricht University, Maastricht, The Netherlands

5 Maastricht Brain Imaging Center, Maastricht University, Maastricht, The Netherlands

6 Brain Imaging Network, University of Coimbra, Coimbra, Portugal 